\title{
The Impact of a New App Channel on Physicians' Performance: Evidence From Online Healthcare Natural Experiment
}

\author{
Weiwei Sun \\ School of Management \\ Harbin Institute of Technology \\ sunweiwei45@126.com
}

\author{
Yuanyuan Dang \\ School of Management \\ Harbin Institute of Technology \\ dyy hit@163.com
}

\author{
Dr.Xitong Guo \\ School of Management \\ Harbin Institute of Technology \\ xitongguo@ hit.edu.cn
}

\begin{abstract}
Besides the web browser, the introduction of the mobile app in online healthcare systems has resulted in an additional touchpoint for users. Drawing on the Media Richness theory, we aim to reveal the effect of the mobile app channel on physicians' performance in the online health communities (OHCs). We provide direct empirical evidence on a large-scale dataset from one of the largest Chinese OHCs, Haodf, and propose a natural experiment to show the casual effect. Our results demonstrate that the introduction of the app channel to OHCs for patients has a positive impact on physicians' responses and rating performance on the online platforms, especially for male physicians from highranking hospitals.
\end{abstract}

\section{Introduction}

With the rapid increase in the number of online health communities (OHCs), such health websites have become one of the primary sources for the public to learn about health and illness [1]. According to Shaheen [2], the appearance of OHCs has transformed the way people obtain health information from traditional physicians' visits to an online search to directly link with consulting doctors in OHCs.

Recently, the growing ubiquity of mobile devices such as smartphones and tablets has spurred the mobile app into becoming another favorite channel for $\mathrm{OHC}$ visits beside the traditional web browsers. Between 2015 and 2016, the global use of mobile devices increased by 429 million (a growth of $5.6 \%$ in a year) with smartphones accounting for most of the growth [3]. Furthermore, over 30,000 health apps were made available in 2013 and this number had grown to 150,000 in 2015 [4]. Based on the fact that the app has become increasingly popular in the online health market, it is imperative for the medical firms on the Internet to become aware of the change in user's behavior and to understand the implications of this change in OHCs.

Much research over the past few decades indicates that adding a new channel have a positive effect on the performance of a business and on individuals' behavior. For instance, a large volume of prior research has focused on the retail market and attempted to reveal how a new retail channel impacts an existing retail channel, especially regarding whether the online channel has complementarity and substitution effects on an existing channel [5-9]. Past studies have also examined the impact of the release of a new channel in the news [10], healthcare [11, 12] and travel markets [13]. However, the causal impact of the mobile app for patients on the physicians' performance in online social health networks has not been thoroughly examined and understood. On the other hand, compared to the web channel, the app channel is also seen to have some weaknesses. According to Chae [14] et al., the general smaller screen size of the mobile device will be inconvenient when a user is using the navigation and input abilities and will make it harder for patients to locate the useful information and choose a suitable physician. On its part, the PC, with its larger screen size will enable users to maintain a comprehensive perspective on the screen and thus enhance the users' decision making. Furthermore, the patient users of the OHCs may avoid using an app channel because they are already similar to the web channel and don't want to spend time on the mobile channel. Accordingly, whether the addition of the app channel for patients a positive impact on physicians' performance does have remains an empirical question.

Despite the increasingly important role of mobile devices in online health communities, how helpful when introducing a new channel to OHCs is largely unknown. Thus, the objective of this study is to examine the causal impact of app channel in online healthcare communities on physicians' performance. In this study, we attempt to address the following research question: What are the effects of introducing a new app channel on physicians' 
responses to patients' questions in terms of quantity and their rating? Also, physicians' online performance varies significantly with their offline status (e.g., working hospital, gender and professional titles), which is especially obvious in our Chinese research context. Physicians with higher professional knowledge are scarcity and care more about reputation in China. This leads to our second research question: How does the effect of multi-channel vary across physicians of different status in online healthcare platforms? We take advantage of an exogenous change in an online health consultation platform and employ a natural experimental research design. The web launched from 2006 and in February 2011 the platform released the mobile app channel. We collect the monthly panel data and use the difference in difference method combine with propensity score matching (PSM) to calculate the effect. Our results show that a new app channel will significantly increase the physicians' performance both in the response and the rating.

The possible mechanism through which a new app channel may facilitate a physician's performance is the Media Richness Theory (MRT). We posit that the mobile app is a richer channel than a web channel in the online health context because the design for the mobile app is more suitable for supporting a medium which can handle multiple information cues simultaneously, such as voice messages. So the mobile app channel, which is richer than a web channel, may have advantages such as delivering richer information within a time interval and enabling real flexible communication between patients and physicians in the health community.

Different users on the same platform may have different reflections on the release of a new channel. According to Jiao $\mathrm{Xu}$ [10], the introduction of a mobile app channel leads to a significant increase in demand at the corresponding mobile news website, and the different users will show different responses to the new app channel.

Our findings on the petitions' performance suggest that the effectiveness of introducing app channel to an online health community. Different physicians' characteristics and the impact of the mobile app channel have different performance. We found that the male physicians have more positive reflections than the female physicians to the introduction of the patients' app channel. The physicians from high-ranking hospitals have positive effects on the app channel.

The rest of the paper is organized as follow. In Section 2, we explore related literature on a multichannel and the Media Richness Theory. Section 3 provides an overview of our research context, experiment design, and the data, while Section 4 describes the empirical model used in our research. In Section 5, we provide the empirical results and discuss the output from the robustness checks. In Section 6, we discuss the study implications and suggestions for future research.

\section{Related Literature and Theory Development}

\subsection{The Multichannel Effect}

There has been a considerable volume of research on assessing the effect of adding a new channel to an existing channel by means of empirical methods $[11,13$, 15, 16]. This stream of research has contributed considerably to revealing the impact of adding an online channel to brick and mortar retail channels [5-7, 9]; on the impacts of adding an offline channel on an existing online retail channel $[8,17]$; on the relation between location-based mobile advertising and offline purchases $[18,19]$; and the impacts of the introduction of a new mobile device channel on an existing channel [10, 20, $21]$. Based on these findings, the prior researchers aimed at addressing the issue of whether a firm should add a new channel in different contexts.

Based on these previous studies, we observe that, in general, whether an online channel is added to an offline channel [11]; or whether an offline channel is added to an online channel, the new channel has a positive effect on the profits of firms and the welfare of customers in the retail market. The introduction of a new Internet channel can spur an enterprise into achieving greater gains in terms of financial performance while the impact is more significant among the powerful firms with a few direct channels [15]. Moreover, the continued presence of the retail stores can result in a short-term decrease in sales only in the catalog channel but can cause a longterm increase in the sales in both the catalog and online channels. On the other hand, it has to be emphasized that adding an e-channel does not always result in benefits [6]; i.e., the users' reactions to a new channel varies considerably across underlying market environments and channel structures [5].

Although previous research has demonstrated the various impacts of the introduction of an app channel to the performance of existing channels, firms, and the individuals. However, there is limited research on the causal impact of the introduction of a mobile app channel to the online healthcare service market. To bridge this study gap, we aim to explore the differences in the physicians' performance before and after the introduction of the patients' app channel and to explore how the impact changes with different physicians, for example in gender, hospital ranking, professional title and online experience in Haodf. 


\subsection{The Media Richness Theory}

The Media Richness theory (MRT) was initially presented by Daft and Lengel [22] as an extension of the information processing theory and the contingency theory. By defining the concept of media richness as "the ability of information to change understanding within a time interval", Daft and Lengel [22], aimed to create a tool to evaluate the richness of communication media such as videos, phone calls, and emails within organizations. The main thrust of the MRT is that a richer communication medium is generally more efficient than leaner mediums for solving equivocal issues. Furthermore, to assist managers to decide whether a medium is rich, Daft [23, 24], gave four characteristics of a rich medium: (1) the ability to handle multiple information cues simultaneously; (2) the ability to facilitate rapid feedback; (3) the ability to establish a personal focus; and (4) the ability to utilize natural language.

After the introduction of the MRT, many communication scholars tried to test and improve on the theory through various kinds of organizational processes and management tasks [25-30]. For example, Sheer [26] indicated that in the process of choosing a communication medium, the managers do not only focus on efficiency but do also consider other factors such as maintenance and relationship growth. On his part, King [31] tried to find another method of evaluating the differences among communication media by introducing social presence, which is a concept for measuring the ability to convey the actual presence of the communication media users. Furthermore, although the MRT was originally presented in the management field, it has been applied to the disciplines outside organizational communication, including distance learning [32-34], civic engagement [35, 36] and healthcare [37-39]. After testing the MRT in various contexts, this theory has been widely adopted and is used as a useful theory to guide managers to choose the right communication media.

One field of research uses the MRT to explain the impact of the richer media on participants' performance and the decision quality. According to Shepherd [32], the students' satisfaction will increase with the improvement of the communication technology's richness. Moreover, Perrault $\left[38^{]}\right.$, also indicated that during the process of choosing a physician in a healthcare platform, a richer medium such as the video can significantly reduce the uncertainty and help patients to make a better decision. Based on these previous findings of the MRT, we accept that adding a new app channel for patients which is richer than a web channel in the e-health context will positively impact physicians' performance in OHCs.

\section{Empirical Background and Data Description}

Our empirical research is based on a large archival dataset of online consultation records and physicians' information from Haodf, one of the largest OHCs in China. Since the establishment of the online platform in 2006, Haodf has become China's leading Internet medical platform. By March 2017, more than 490,000 physicians from 7,500 hospitals had participated on this platform. Furthermore, the online consultations in this platform had peaked to over 15 million by May 2018, implying that Haodf had become a popular large $\mathrm{OHC}$ in China.

On the Haodf platform, the patients can choose a physician for consultation based on the description and reviews of physicians and then the communication was just between them but the consultation can be seen by others. All the messages from the patients and physicians will be recorded with the time recorded in the question page. We collected all the available online consultation data between February 2010 and February 2012 directly from the Haodf website by web spider to ensure the authenticity of the records. Our data consists of 5.3 million questions asked by the patients on the platform, and about 25,638 physicians had answered these questions during the observation period.

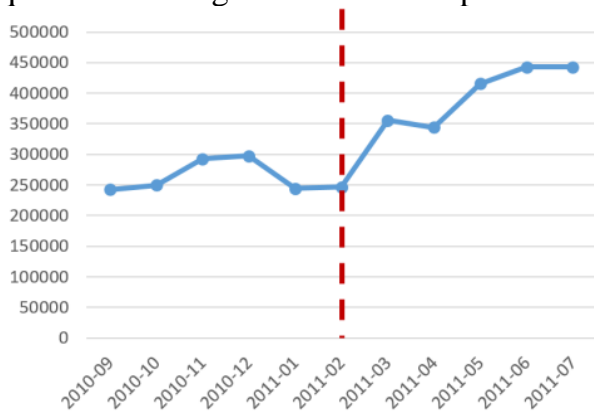

Firugre 1. Number of Physicians' Responses Over Time

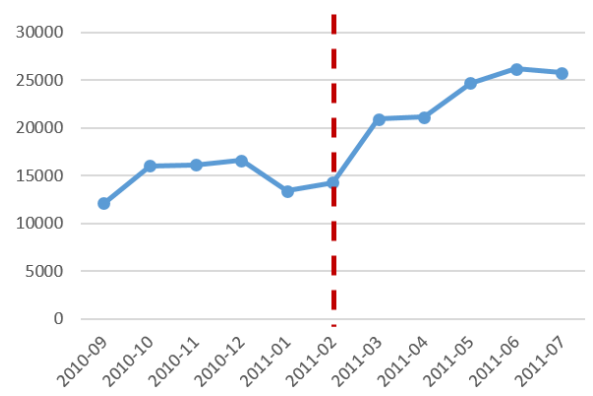

Firugre 2. Number of Physicians' Online Gifts Over Time

We use physicians' responses number and online gifts number to measure their response performance and rating performance respectively. From Figure 1 and 2, 
we can find that both the physicians' response number and online gift number increased more steeply after the launch of the app.

In order to measure the actual impact of the new app channel for patients on the physicians' performance, we pick the active physicians who had registered with their legal names before the release of the app channel as our research samples. Among the active physicians, 2,364 physicians (treated group) had ever responded to the patients' messages which come from the app channel and 15,219 physicians (control group) hadn't. In order to make sure the control and treated are comparable in terms of observable characteristics, we use propensity score matching (PSM) to create a "proper" control group for treated physician. We matching each treated physician to the most "similar" control one in terms of: (i) physician characteristics, such as their gender (=1:female; $=2$ :male; $=3:$ missing value), hospital level (=1:low; =2:high; $=3$ :missing value), professional title (=1-4:low-high; =5:missing value) and province; and (ii) their experience in Haodf, which is the moths from the opening of their home page in Haodf to the introduction of the app channel. Then, we use $t$-test to assess the quality of matching and the results are presented in Table 1 . We can see that the covariate imbalance has been greatly reduced after the matching.

The main statistical concern for a natural experiment is usually to identify the actual impact of the treatment and exclude the effects from the selection bias. In our research, we aimed to ensure that the difference in the physicians' performance is the result of the effects of the new app channel for patients. While we could observe from the descriptive statistical analysis, the impact of the mobile app channel generally, however, this graphical result might be influenced by some un observed system biases which are related to app channel usage. Fortunately, the selection bias was not a key problem in our research context because the physicians in the Haodf platform could not identify whether this message had come from the app channel, and hence, the physicians randomly set into control or treatment group.

Table 1. Differences in Mean Before and After Matching

\begin{tabular}{ccccccccccc}
\hline & \multicolumn{4}{c}{ Before Matching } & \multicolumn{4}{c}{ After matching } \\
\hline \multirow{2}{*}{ Variables } & $\begin{array}{c}\text { Mean } \\
\text { Treated }\end{array}$ & $\begin{array}{c}\text { Mean } \\
\text { Control }\end{array}$ & \%bias & $\begin{array}{c}t- \\
\text { statistics }\end{array}$ & $\begin{array}{c}p \text { - } \\
\text { value }\end{array}$ & $\begin{array}{c}\text { Mean } \\
\text { Treated }\end{array}$ & $\begin{array}{c}\text { Mean } \\
\text { Control }\end{array}$ & \%bias & $\begin{array}{c}t- \\
\text { statistics }\end{array}$ & $\begin{array}{c}p- \\
\text { value }\end{array}$ \\
\hline gender & 1.7923 & 1.8639 & -14.0 & -6.11 & 0.000 & 1.7923 & 1.805 & -2.5 & -0.92 & 0.360 \\
hospital & 2.0102 & 1.946 & 15.7 & 6.41 & 0.000 & 2.0102 & 2.008 & 0.5 & 0.21 & 0.833 \\
title & 3.5051 & 3.1825 & 42.6 & 17.78 & 0.000 & 3.5051 & 3.5085 & -0.4 & -0.18 & 0.860 \\
province & 9.8439 & 12.541 & -28.8 & -13.01 & 0.000 & 9.8439 & 9.7276 & 1.2 & 0.43 & 0.668 \\
experience & 19.109 & 15.272 & 39.5 & 17.73 & 0.000 & 19.109 & 19.06 & 0.5 & 0.17 & 0.863 \\
\hline
\end{tabular}

\section{Econometric Model}

The treatment effect of introducing app channel on the physician's performance in our experimental setup is identified by infering their counterfactual response and rating in the post-app introduced period from the response number and online gifts number of unaffected physicians in the period. Thus, for clean identification of treatment effect, the effect of all observed and unobserved factors, other than introducing a new app channel, on performance of affected and unaffected physicians should be similar. It is worth noting that the PSM can only resolve biases from the observed characteristics, so we also use the difference in difference (DID) method to exclude the biases from the unobserved factors which are stable overt time in their influence on the physicians' performance. Our main estimating model for the physician $i$ in month $t$ is:

$$
\begin{aligned}
& \text { Outcome }_{i t}=\alpha \times \text { Treatment }_{i} \\
& +\beta \times \text { AfterTreatment }{ }_{t} \\
& +\gamma \times \text { Treatment }_{i} \\
& \times \text { AfterTreatment }_{t} \text { ) } \\
& +k_{i}+\varepsilon_{i t}
\end{aligned}
$$

where the Outcome $_{i t}$ is the online gifts number and the logarithmic transformation of response number of the

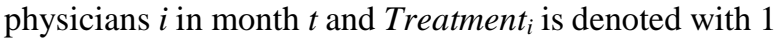
if the physician $i$ has got the patients' messages which come from the mobile app of the Haodf platform and 0 if otherwise. AfterTreatment ${ }_{i t}$ is a dummy variable that indicates the post-app introduction period for both the treated group and the control group. For example, whether a physician belongs to the control group or the treated group, this variable will be 1 , in the month after February 2011 and will be 0 for February 2011 and the months before. The coefficient $\beta$ for the interaction term in the Model 1, Treatment $_{i} \times$ AfterTreatment $_{i t}$, represents the DID estimators that can capture the difference in the physicians' performance (responses and rating) in the treated group after the release of the app in contrast to the difference in the physicians in the control group during the same period.

In addition to these variables, we also controlled for the observed covariables, $k_{i}$, which includes some features of the physicians such as gender, the level of their professional title, the level of their hospital, their department, their province and their experience of 
Haodf. By the way, when the Outcome it is response number, we also add the question number, which is the number of questions replied by physicians, for it is the most important factor related to the response number. We used these covariables to control the observed individual difference across the physicians' performance. In addition to this model, we also used the individual fixed effect and time fixed effect to more accurately estimate the impact of the app channel on the physicians' performance in the treated group. The model for the physician $i$ in month $t$ is:

Outcome $_{i t}=\alpha \times$ Treatment $_{i}$ $+\beta \times$ AfterTreatment $t_{t}$ $+\gamma \times$ Treatment $_{i}$ $\times$ AfterTreatment $\left.t_{t}\right)$ $+x_{i}+\varphi_{t}+k_{i}+\varepsilon_{i t}$

In this model, we added the $x_{i}$ to control the related variables which were only related to the individual but not to time such as the gender and the $\varphi_{t}$ to control the variables which only changed across time but not the individual such as the holiday and the season. Compared with Model 1, this model can provide more accurate estimators for the impact of the mobile app channel.

To explore the question of whether the different kinds of physicians indicate varied reflections on the new app channel and whatever differences there would be, we built the new model as follows:

Outcome $_{i t}=\alpha \times$ Treatment $_{i}$ $+\beta \times$ AfterTreatment ${ }_{t}$ $+\gamma \times$ Treatment $_{i}$ $\times$ AfterTreatment $\left._{t}\right)$ $+\lambda \times$ (Treatment $_{i}$ $\times$ AfterTreatment ${ }_{t} \times k_{i}$ ) $+x_{i}+\varphi_{t}+\varepsilon_{i t}$ professional title, hospital's rank and their experience in Haodf. Based on Model 2, we added a new term, Treatment $_{i} \times$ AfterTreatment $t_{i t} \times k_{i}$, and used the coefficient $\lambda$ for the term to represent how the $k_{i}$ impacted the ATT. In this model, we could reveal how the physicians' features moderated the ATT.

\section{Empirical Results}

\subsection{Main Results}

Table 2 shows the estimated coefficients of the main results of our research. The first and the third columns in Table1 present the regression results of Model 1 for the log-transformed response numbers and gift number of the physicians on the Haodf platform. We applied the widely adopted DID model to capture the ATT, which represents the average effect of the patients' mobile app channel for the physicians who had responded to the app channel's messages in the consultations of patients during the 24-month research period. In Column 1, the estimated coefficient of Treatment ${ }_{i} \times$ AfterTreatment $_{i t}$ is 0.107 and indicates that compared with the physicians in the control group, these physicians in the treated group would response about $11 \%$ more after the introduction of the app. Similarity, the coefficient is 3.875 in column 3 which means the treated physicians would get about 4 more online gifts for the introduction of app channel. Based on the results in Column 1 and 3, we can postulate that the introduction of the app channel really motivated the physicians in the treated group to render more responses and help them get more online gifts per month.

$k_{i}$ represents the four features of the physicians: gender,

Table 2. Main Estimation Results

\begin{tabular}{lcccc}
\hline & $(1)$ & $(2)$ & $(3)$ & $(4)$ \\
& lrep_num & lrep_num & gifts & gifts \\
\hline Treat*After & $0.107^{* * *}$ & $0.122^{* * *}$ & $3.875^{* * *}$ & $3.930^{* * *}$ \\
& $(0.004)$ & $(0.003)$ & $(0.085)$ & $(0.058)$ \\
\hline Control & $\sqrt{ }$ & $\sqrt{ }$ & $\sqrt{ }$ & $\sqrt{ }$ \\
Time FE & & $\sqrt{ }$ & & $\sqrt{ }$ \\
Individual FE & & 0.157 & 0.900 & $-2.607 * * *$ \\
\hline Constant & 0.005 & $(0.103)$ & $0.668)$ & $(0.618)$ \\
& $(0.026)$ & 0.984 & 0.137 & 0.609 \\
\hline R-squared & 0.973 & 86569 & 86569 & 86569 \\
$\mathrm{~N}$ & 86569 & & &
\end{tabular}

To obtain an accurate estimator for the ATT, we tried to control the potential biases more meticulously by adding the $x_{i}$ and the $\varphi_{t}$ to the model. In Model 2, the $x_{i}$ represents the individual fixed effect and it can control the relevant features which are only related to the individual. Moreover, the $\varphi_{t}$ represents the time fixed effect in this model and thus we can exclude the biases 
from the unobserved variables which are related to the time taken.

The regression results of Model 2 are presented on the second and the fourth columns of Table 2. Column 2 includes a model which control the relevant variables which may influence the impact of the app channel. In comparing the results in Column 1, the estimated coefficient of Treatment $_{i} \times$ AfterTreatment $_{i t}$ is 0.122 which means that we might have isolated some unobserved variables that can lead to the underestimation of the app channel's impact in Column 1. By comparing the standard error between Columns 1 and 2, we found that the standard error in Column 2 is smaller. The above two phenomenon are found to be the same between Columns 3 and 4. Accordingly, we can verify that the estimated coefficients in Model 2 which use the time fixed effect and the individual effect are more accurate.

In summary, the positive and statistically significant coefficients $(\mathrm{p}<0.01)$ in our above empirical analysis strongly support the postulations that the introduction of patients' app channel can improve the response performance and rating performance of physicians on the platform.

\subsection{Robustness Checks}

To further verify that the estimators of the above empirical analysis are robust, we used several methods to check the results.

As the first robustness check, we established various new variables in Model 2 to measure the response performance, including the following: (1) the number of questions to which a physician responded; (2) the total length of replies content of a physician; The Columns 14 in Table 3 respectively represent the two variables. In our specifications, the length of responses is logtransformed to obtain the impact of the app to the growth rate of the length of the response. In the first column of Table 3, the coefficient of Treatment $_{i} \times$ AfterTreatment $_{i t}$ is 0.242 , and the coefficient increased a little after adding the fixed effect in Column 2. These significant $(p<0.01)$ estimators demonstrate that the physicians in the treated group tend to deal more questions than the physicians in the control group after the introduction of the app.

We observe the different situation in in Columns 3 and 4 of Table 3 . The coefficient in Column 3 indicates that the total length of the monthly replies decreased about $10 \%$ after the launch of the app channel, and the coefficient is even smaller with the fixed effect in Column 4. By the way, we also add the response number, which is very related to the response length, as one of the control variables in Column 3 and 4 . The above results indicate that the total length of response significant $(p<0.01)$ decreased for the physicians in treated group after app introduction.

Considering the above two results, it seems that the physicians in treated group deal more questions with shorter reply after the introduction of the app channel. I believe this can provide evidences to support the above result that the app channel for patients have a positive impact on the physicians' response performance.

To further prove the app channel has a positive impact on physicians' rating performance, we use another rating value which is the number of the online thanks letter as our dependent variable in the second robustness check. The regression results are presented in Columns 5 and 6 of Table 3. The estimated coefficients of the term, Treatment $_{i} \times$ AfterTreatment $_{i t}$ in Column 5 demonstrates that after the introduction of the app, the thanks letter number of the treated physicians increased 0.204. In Column 6, the coefficient of the same term is $0.226(\mathrm{p}<0.01)$ after adding the fixed effect. The above results indicate that the physicians in treated group receive more online thanks letters after the introduction of the mobile app.

In summary, based on the above analysis, we perceive that physicians' response and rating performance show significant improvement after the introduction of the patients' mobile app and this confirms the robustness of our empirical results.

Table 3. Robustness Check Results

\begin{tabular}{|c|c|c|c|c|c|c|}
\hline & $\begin{array}{c}\text { (1) } \\
\text { lque_num }\end{array}$ & $\begin{array}{c}(2) \\
\text { lque_num }\end{array}$ & $\begin{array}{c}(3) \\
\text { llength }\end{array}$ & $\begin{array}{c}(4) \\
\text { llength }\end{array}$ & $\begin{array}{c}(5) \\
\text { thanks letter }\end{array}$ & $\begin{array}{l}(6) \\
\text { thanks letter }\end{array}$ \\
\hline Treat*After & $\begin{array}{c}0.242^{* * * *} \\
(0.019) \\
\end{array}$ & $\begin{array}{c}0.263 * * * \\
(0.013) \\
\end{array}$ & $\begin{array}{c}-0.103 * * * \\
(0.020) \\
\end{array}$ & $\begin{array}{c}-0.202 * * * \\
(0.016) \\
\end{array}$ & $\begin{array}{c}0.204 * * * \\
(0.009)\end{array}$ & $\begin{array}{c}0.226 * * * \\
(0.007)\end{array}$ \\
\hline $\begin{array}{l}\text { Control } \\
\text { Time FE } \\
\text { Individual FE }\end{array}$ & $\sqrt{ }$ & $\begin{array}{l}\sqrt{ } \\
\sqrt{ } \\
\sqrt{ }\end{array}$ & $\sqrt{ }$ & $\begin{array}{l}\sqrt{ } \\
\sqrt{ } \\
\sqrt{ }\end{array}$ & $\sqrt{ }$ & $\begin{array}{l}\sqrt{ } \\
\sqrt{ } \\
\sqrt{ }\end{array}$ \\
\hline Constant & $\begin{array}{c}0.768 * * * \\
(0.112) \\
\end{array}$ & $\begin{array}{c}-1.032 * * \\
(0.468) \\
\end{array}$ & $\begin{array}{c}1.102 * * * \\
(0.085) \\
\end{array}$ & $\begin{array}{c}1.577 * * * \\
(0.500) \\
\end{array}$ & $\begin{array}{c}-0.014 \\
(0.048) \\
\end{array}$ & $\begin{array}{c}-0.815 * * * \\
(0.133)\end{array}$ \\
\hline $\begin{array}{l}\text { R-squared } \\
\mathrm{N}\end{array}$ & $\begin{array}{l}0.339 \\
86569\end{array}$ & $\begin{array}{l}0.749 \\
86569\end{array}$ & $\begin{array}{l}0.889 \\
86569\end{array}$ & $\begin{array}{l}0.936 \\
86569\end{array}$ & $\begin{array}{c}0.103 \\
86569\end{array}$ & $\begin{array}{l}0.445 \\
86569\end{array}$ \\
\hline
\end{tabular}

$* \mathrm{p}<0.10, * * \mathrm{p}<0.05, * * * \mathrm{p}<0.010$ 
Table 4. Influence of Physicians' Characteristics for the Impact on an App Channel

\begin{tabular}{|c|c|c|c|c|}
\hline & $\begin{array}{c}\text { (1) } \\
\text { lrep_num }\end{array}$ & $\begin{array}{c}\text { (2) } \\
\text { lrep_num }\end{array}$ & $\begin{array}{c}(3) \\
\text { gifts }\end{array}$ & $\begin{array}{c}(4) \\
\text { gifts }\end{array}$ \\
\hline Treat*After & $\begin{array}{c}0.071 \\
(0.070)\end{array}$ & $\begin{array}{c}0.161 * * * \\
(0.060)\end{array}$ & $\begin{array}{c}2.770 \\
(1.999)\end{array}$ & $\begin{array}{c}4.131 * * \\
(1.862)\end{array}$ \\
\hline Gender*Treat*After & $\begin{array}{c}0.019 * * * \\
(0.007)\end{array}$ & $\begin{array}{c}0.013 * * \\
(0.006)\end{array}$ & $\begin{array}{c}1.607 * * * \\
(0.138)\end{array}$ & $\begin{array}{c}1.422 * * * \\
(0.110)\end{array}$ \\
\hline Hospital*Treat*After & $\begin{array}{c}0.064 * * * \\
(0.011)\end{array}$ & $\begin{array}{c}0.076 * * * \\
(0.010)\end{array}$ & $\begin{array}{c}2.022 * * * \\
(0.226)\end{array}$ & $\begin{array}{c}2.058 * * * \\
(0.175)\end{array}$ \\
\hline Experience*Treat*After & $\begin{array}{c}0.001 * * * \\
(0.000)\end{array}$ & $\begin{array}{c}-0.001 * * * \\
(0.000)\end{array}$ & $\begin{array}{c}0.095 * * * \\
(0.008)\end{array}$ & $\begin{array}{c}0.066^{* * *} * \\
(0.006)\end{array}$ \\
\hline Title_2*Treat*After & $\begin{array}{l}-0.081 \\
(0.070)\end{array}$ & $\begin{array}{c}-0.119 * * \\
(0.060)\end{array}$ & $\begin{array}{c}-5.793 * * * \\
(1.985)\end{array}$ & $\begin{array}{c}-6.428 * * * \\
(1.857)\end{array}$ \\
\hline Title_3*Treat*After & $\begin{array}{l}-0.053 \\
(0.070)\end{array}$ & $\begin{array}{c}-0.098 * \\
(0.059)\end{array}$ & $\begin{array}{c}-3.993 * * \\
(1.985)\end{array}$ & $\begin{array}{c}-4.725 * * \\
(1.855)\end{array}$ \\
\hline Title_4*Treat*After & $\begin{array}{c}-0.051 \\
(0.070)\end{array}$ & $\begin{array}{c}-0.095 \\
(0.059)\end{array}$ & $\begin{array}{l}-3.363 * \\
(1.984)\end{array}$ & $\begin{array}{c}-4.118 * * \\
(1.854)\end{array}$ \\
\hline $\begin{array}{l}\text { Control } \\
\text { Time FE } \\
\text { Individual FE }\end{array}$ & $\sqrt{ }$ & $\begin{array}{l}\sqrt{ } \\
\sqrt{ } \\
\sqrt{ }\end{array}$ & $\sqrt{ }$ & $\begin{array}{l}\sqrt{ } \\
\sqrt{ } \\
\sqrt{ }\end{array}$ \\
\hline Constant & $\begin{array}{c}0.009 \\
(0.017)\end{array}$ & $\begin{array}{c}-0.104 * * * \\
(0.033)\end{array}$ & $\begin{array}{c}1.041 * * * \\
(0.215)\end{array}$ & $\begin{array}{c}-0.101 * * * \\
(0.014)\end{array}$ \\
\hline $\begin{array}{l}\text { R-squared } \\
\mathrm{N}\end{array}$ & $\begin{array}{c}0.973 \\
86569\end{array}$ & $\begin{array}{c}0.984 \\
86569\end{array}$ & $\begin{array}{l}0.144 \\
86569\end{array}$ & $\begin{array}{l}0.446 \\
86569\end{array}$ \\
\hline
\end{tabular}

\subsection{Physicians' Characteristics Impacting the App Channel}

We have demonstrated that the patients' new app channel had a significant positive impact on the physicians' performance through a series of empirical analysis and robustness checks of the main results. Next, we focused on exploring whether different types of physicians have various reflections on the new app channel. In their study, Jiao Xu [10], indicated that different types of consumers exhibit various levels of complementarity between web channels and app channels in the news market. We can assume that the characteristics of physicians may also influence the impact of the mobile app and we explored this question in Model 3. The regression results of Model 3 are presented in Tables 4.

In the first and second column of Table 3, we explore whether the characteristics of physicians can influence the impact of the mobile app for patients on physicians' response performance. From the coefficient of the term, Gender $_{i} \times$ Treatment $_{i} \times$ AfterTreatment $t_{i}$, we perceive that, generally, the male physicians tend to have a more positive reflection on the new app channel compared with female physicians. Similarity, the coefficient of Hospital $_{i} \times$ Treatment $_{i} \times$ AfterTreatment $_{i t}$, in Column 3 shows that the physicians from different ranks of the hospitals have a significant difference $(p<0.01)$ in their reflection on the patients' app channel. In fact, the physicians from a high-ranking hospital, namely a Grade-A Tertiary Hospital, have a more positive reflection towards the patients' new app channel than the physicians from low-ranking hospitals. From the coefficient of Experience $_{i} \times$ Treatment $_{i} \times$ AfterTreatment ${ }_{i t}$, we can see that although this estimator is significant $(\mathrm{p}<0.01)$, the influence of physicians' online experience in Haodf is small. Finally, we observe that the title $_{i}$ is not a statistically significant variable which indicates that the job title of physicians does not have a significant influence on the impact of the app channel in our research samples.

Compared with Column 1, several differences appeared after adding the fixed effect in Columns 2: (i) the impact of physicians' online experience become negative but the impact is still small; (2) the title of physicians has a significant $(\mathrm{p}<0.01)$ negative influence on app channel's impact.

Equally, in Column 3 and 4, we explore how the characteristics of physicians influence the impact of the mobile app for patients on physicians' rating performance. Generally, the Gender, Hospital, Experience $_{i}$ are all have positive influence on the app channel's impact but the title $e_{i}$ is the difference. From the estimated coefficients, we can see that the high title level has a significant $(\mathrm{p}<0.01)$ negative influence on the 
impact of app channel compared with low one and the higher the title level, the smaller the influence.

\section{Discussion}

To explain our results, we introduced the Media Richness Theory which was created by Daft and Lengel [22]. This theory indicates that different types of communication media have various information richness which is defined as "the ability of information to change understanding within a time interval", and the richer communication mediums are generally more effective for dealing with equivocal issues [22]. In our research context, we believe that the positive effect of the new app channel is because the new app channel is richer than existing web channels from two aspects: (1) the ability to facilitate rapid feedback, and (2) the ability to handle multiple information cues simultaneously.

Firstly, patient users can provide feedback more rapidly through a mobile app than web browser because the apps are installed in the mobile devices which are more portable than others devices. By the way, the mobile app always reminds you if you receive a message but the web browser doesn't. Accordingly, the app channel has more advantages to support rapid feedback. Secondly, in our research context, the voice message which is a richer medium than a text, can only be sent from an app channel and the other media such as the phone and text messages are supported in both channels, Thus, we can postulate that the app channel has advantages in its ability to handle multiple information cues simultaneously.

In brief, we assert that the app channel is a richer channel than a web channel in our research context, and hence the introduction of app channel for patients can result in a positive impact on the performance of physicians on the OHCs.

Moreover, the empirical results show that characteristics of physicians can moderate the app's impact on physicians' performance. Compared with female physicians, the male physicians' performances are more positively affected by the app channel. The finding that physicians from high-ranking hospitals have a more positive reflection on the introduction of patients' app channel means these physicians prefer to replying more to the patients and receive more online gifts compared with others. A possible reason is that the high-ranking hospitals are mainly located in the developed cities and the physicians in these cities are more likely to be encouraged by richer media. We also find the online experience can positively influence the impact of the mobile app for patients on physicians' rating performance but it is not very important on physician response performance. The same phenomenon can also be seen in the influence of title, the title can negatively influence the app channel's impact on physicians rating but not on their response performance.

\subsection{Managerial Implications}

The appearance of the OHCs is an important innovation in the development of e-health, and it can provide a reliable source for users to get health information. The rapid spread of the mobile Internet has enriched the development of the app and there is an increasing number of Chinese online health community have their own mobile app for users. Based on the phenomenon, it is necessary for the health firms to know whether the mobile app channel for patients to the health platform can bring positive impacts and how the behavior of the physicians' change after the introduction of a new app channel.

Our findings have several managerial implications for the managers of OHCs in understanding the impact of a new app channel for patients on the physicians' performance and deciding whether an online health platform should add an app channel to an existing web channel. In our research context, we can state formally that adding a new app channel for patients to the $\mathrm{OHC}$ can improve physicians' performance, and the impact will be even more positive for male physicians from high-ranking hospitals. Based on this finding, if the manager of an online health community wants to add an app channel, he should consider the structure of the users on the platform in order to get a better effect.

\subsection{Limitations and Suggestions for future Research}

Our research has two main limitations. Firstly, we believe that response performance is a complex variable which is difficult to measure. In our research, we mainly used the number of responses to measure the physicians' response performance; however, the response performance of the physicians does not only involve its quantity but also the quality. It is possible that a physician renders many responses but the quality for each response may not be high and this situation cannot be distinguished in our research context. Secondly, because we collected the data from the OHCs directly instead of getting the data from the databases of the OHCs, we have some limitations in our data such as the lack of complete demographic information of the physicians. Because of the data restrictions, we failed to explore how other characteristics of physicians influence the ATT in our research.

Accordingly, we suggest that future research can focus in two directions. Firstly, in the future, researchers can measure response performance from different 
aspects and can even build a series of measurement standards on the response performance of physicians in the OHCs. An alternate way of measuring the performance of users is by analyzing the message content using a machine learning method and a natural language process. Secondly, a future research project can focus on other types of users in the OHCs which is the patient-user, and examine how the introduction of a patients' app channel affects the patients' behavior on the platform.

\section{Conclusion}

In this paper, we focused on the question about how the new app channel for patients impacted the physicians' response and rating performance of a Chinese online health community. After reviewing the existing studies, we verify that many researchers have tried to reveal the influence of the introduction of an app channel to existing channels and the users in various research fields such as the news, medical, and travel markets. However, we have not seen any research exploring the relationship between the launch of the app channel for patients and the physicians' performance in the online health community, especially in the Chinese online health market.

Recently, the online health community has become a popular platform for the patients and physicians to communicate with each other because it can remove temporal and geographical restrictions, and this is why an increasing number of people prefer consulting physicians on the OHCs rather than visiting the physicians' physical clinics in China. Furthermore, a recent survey indicates that the mobile network in China has developed rapidly [3]. Based on the above considerations, we believe our research is meaningful both academically and in practice.

Using large-scale second-hand data from Haodf, which is one of the largest online health communities in China, we hope to reveal the differences in the physicians' response and rating performance on this OHC before and after the introduction of a mobile app channel for patients to the platform. To capture the average treatment effect on the treated (ATT), we applied the difference-in-difference approach combine with PSM in our research, which are widely adopted technology in econometric research. Moreover, because the variable that decide which group (treated group and control group) the physicians belong to is exogenous, accordingly, the samples in the treated group and control group can be compared.

By means of a series of empirical methods and several checks, we have provided robust evidence that the launch of a mobile app channel has a positive impact on physicians' performance on the OHCs. Besides, we also further explored whether different types of physician users on the platform have various reflections on the introduction of an app channel. According to our empirical results, we find that the male physicians have a more positive reaction than the female physicians. Although the physicians' professional title does not influence the app channel's impact on the response but it is import on physicians' rating performance. Similarity, the online experience in Haodf have a positive impact on physicians' rating performance but not on their response performance. Finally, we find the physicians from a Grade-A Tertiary Hospital reflect more positively than the ones from lower level hospitals.

\section{References}

[1] Ziebland S, Chapple A, Dumelow C, et al. How the internet affects patients' experience of cancer: a qualitative study [J]. Bmj, 2004, 328(7439): 564.

[2] Kanthawala S, Vermeesch A, Given B, et al. Answers to health questions: internet search results versus online health community responses [J]. Journal of medical Internet research, 2016, 18(4):

[3] Cisco C V N I. Global mobile data traffic forecast update, 2013-2018 [J]. white paper, 2014,

[4] Elbert S P, Dijkstra A, Oenema A. A mobile phone app intervention targeting fruit and vegetable consumption: the efficacy of textual and auditory tailored health information tested in a randomized controlled trial $[\mathrm{J}]$. Journal of medical Internet research, 2016, 18(6):

[5] Yoo W S, Lee E. Internet channel entry: A strategic analysis of mixed channel structures [J]. Market Sci, 2011, 30(1): 29-41.

[6] Langer N, Forman C, Kekre S, et al. Ushering buyers into electronic channels: An empirical analysis [J]. Information Systems Research, 2012, 23(4): 1212-31.

[7] Biyalogorsky E, Naik P. Clicks and mortar: the effect of on-line activities on off-line sales [J]. Marketing Letters, 2003, 14(1): 21-32.

[8] Wang k, Goldfarb A. Can offline stores drive online sales? [J]. Journal of Marketing Research, 2017, 54(5): 706-19.

[9] Jing B. Showrooming and Webrooming: Information Externalities Between Online and Offline Sellers [J]. Market Sci, 2018,

[10] Xu J, Forman C, Kim J B, et al. News media channels: Complements or substitutes? Evidence from mobile phone usage [J]. Journal of Marketing, 2014, 78(4): 97-112.

[11] Bavafa H, Hitt 1 M, Terwiesch C. The impact of e-visits on visit frequencies and patient health: Evidence from primary care [J]. Management Science, 2018,

[12] Kumar A, Telang R. Does the web reduce customer service cost? Empirical evidence from a call center [J]. Information Systems Research, 2012, 23(3-part-1): 721-37.

[13] Granados N, Gupta A, Kauffman R J. Online and offline demand and price elasticities: Evidence from the air travel industry [J]. Information Systems Research, 2012, 23(1): 16481. 
[14] Chae M, Kim J. Do size and structure matter to mobile users? An empirical study of the effects of screen size, information structure, and task complexity on user activities with standard web phones $[\mathrm{J}]$. Behaviour \& information technology, 2004, 23(3): 165-81.

[15] Geyskens I, Gielens K, Dekimpe M G. The market valuation of internet channel additions [J]. Journal of marketing, 2002, 66(2): 102-19.

[16] Soysal G, Krishnamurthi L. How Does Adoption of the Outlet Channel Impact Customers' Spending in the Retail Stores: Conflict or Synergy? [J]. Management Science, 2015, 62(9): 2692-704.

[17] Avery J, Steenburgh T J, Deighton J, et al. Adding bricks to clicks: Predicting the patterns of cross-channel elasticities over time [J]. Journal of Marketing, 2012, 76(3): 96-111.

[18] Dinner I M, Van Heerde H J, Neslin S A. Driving online and offline sales: The cross-channel effects of traditional, online display, and paid search advertising [J]. Journal of Marketing Research, 2014, 51(5): 527-45.

[19] Fang Z, Gu B, Luo X, et al. Contemporaneous and delayed sales impact of location-based mobile promotions [J]. Information Systems Research, 2015, 26(3): 552-64.

[20] Bang Y, Lee D-J, Han K, et al. Channel capabilities, product characteristics, and the impacts of mobile channel introduction [J]. Journal of Management Information Systems, 2013, 30(2): 101-26.

[21] Xu K, Chan J, Ghose A, et al. Battle of the channels: The impact of tablets on digital commerce [J]. Management Science, 2016, 63(5): 1469-92.

[22] Daft R L, Lengel R H. Organizational information requirements, media richness and structural design [J]. Management science, 1986, 32(5): 554-71.

[23] Lengel R H, Daft R L. The selection of communication media as an executive skill [J]. Academy of Management Perspectives, 1988, 2(3): 225-32.

[24] Daft R L, Lengel R H: Texas A and M Univ College Station Coll of Business Administration, 1983.

[25] Dennis A R, Kinney S T. Testing media richness theory in the new media: The effects of cues, feedback, and task equivocality [J]. Information systems research, 1998, 9(3): 256-74.

[26] Dennis A R, Valacich J S. Rethinking media richness: Towards a theory of media synchronicity; proceedings of the Systems Sciences, 1999 HICSS-32 Proceedings of the 32nd Annual Hawaii International Conference on, F, 1999 [C]. IEEE.

[27] Sheer V C, Chen L. Improving media richness theory: A study of interaction goals, message valence, and task complexity in manager-subordinate communication [J]. Management Communication Quarterly, 2004, 18(1): 76-93.

[28] Brunelle E, Lapierre J. Testing media richness theory to explain consumers' intentions of buying online; proceedings of the Proceedings of the 10th international conference on Electronic commerce, F, 2008 [C]. ACM.

[29] Brunelle E. Introducing media richness into an integrated model of consumers' intentions to use online stores in their purchase process [J]. Journal of Internet Commerce, 2009, 8(3-4): 222-45.

[30] Simon S J, Peppas S C. An examination of media richness theory in product web site design: An empirical study [J]. info, 2004, 6(4): 270-81.
[31] King R C, Xia W. Media appropriateness: Effects of experience on communication media choice $[\mathrm{J}]$. Decision Sci, 1997, 28(4): 877-910.

[32] Shepherd M M, Martz JR W B. Media richness theory and the distance education environment [J]. J Comput Inform Syst, 2006, 47(1): 114-22.

[33] Lan Y-F, Sie Y-S. Using RSS to support mobile learning based on media richness theory [J]. Computers \& Education, 2010, 55(2): 723-32.

[34] Sun P-C, Cheng H K. The design of instructional multimedia in e-Learning: A Media Richness Theory-based approach [J]. Computers \& education, 2007, 49(3): 662-76.

[35] Brinker D L, Gastil J, Richards R C. Inspiring and informing citizens online: A media richness analysis of varied civic education modalities [J]. Journal of Computer-Mediated Communication, 2015, 20(5): 504-19.

[36] Burgess J E, Foth M, Klaebe H G. Everyday creativity as civic engagement: A cultural citizenship view of new media [J]. 2006,

[37] Perrault E K, Smreker K C. What can we learn from physicians' online biographies to help in choosing a doctor? Not much. A content analysis of primary care physician biographies [J]. Journal of Communication in Healthcare, 2013, 6(2): 122-7.

[38] Perrault E K. Patients' preferences for family medicine physicians' video biographies [J]. Health marketing quarterly, 2016, 33(4): 342-52.

[39] Ong L M, De Haes J C, Hoos A M, et al. Doctor-patient communication: a review of the literature [J]. Social science \& medicine, 1995, 40(7): 903-18. 